\title{
Schizophrenia with Somatic Delusions: A Case Report
}

\author{
Rahulkumar Patel ${ }^{1}$, Luisa Gonzalez ${ }^{1}$ and Andrew Joelson ${ }^{2}$, Panagiota Korenis ${ }^{1 *}$ \\ ${ }^{1}$ Department of Psychiatry, Albert Einstein College of Medicine-Bronx Lebanon Hospital Center, New York, USA \\ ${ }^{2}$ Albert Einstein College of Medicine, USA
}

\begin{abstract}
Schizophrenia is a mental illness that affects approximately $1 \%$ of the general population. Symptoms most commonly include auditory hallucinations, several types of delusions, disorganization in speech and behavior, formal thought disorder, and negative symptoms including poverty of speech, thought or motivation. Of the delusional symptoms, somatic delusions-those that pertain to the body-are rather rare. Somatic delusions are defined as fixed false beliefs that one's bodily function or appearance is grossly abnormal. They are a poorly understood psychiatric symptom and pose a significant clinical challenge to clinicians. Studies indicate that only one third of this patient population has a positive treatment outcome with resolution of symptoms. While challenges exist in treating patients when they present with somatic delusions alone, it becomes far more difficult when somatic delusions present in patients with schizophrenia. Physical symptoms such as pain or discomfort are often incorrectly perceived or misinterpreted by psychotic patients. Often medical conditions are overshadowed by psychosis and may get undiagnosed or overlooked which could potentially result in fatal errors for patients. While little has been written about patients with true delusions that are somatic in nature, somatic delusions, specifically somatic delusions associated with schizophrenia are particularly under reported in the literature. Here, we describe the case of a 40-year-old Hispanic-American patient with established chronic schizophrenia. From her initial presentation to the hospital, she exhibited and perseverated on a number of somatic delusions about her body including her bones being "twisted" around one another, delusions of pregnancy and abortion, and her normally-functioning arm being broken. This paper will also explore treatment challenges posed by these complicated patients. In addition, a review of potential cultural influences as well as the economic burden that such patients place on the health care sector by their numerous emergency room and office visits will be discussed.
\end{abstract}

Keywords: Somatic delusions; Schizophrenia; ER visits

\section{Introduction}

The definition of schizophrenia as a disease entity has undergone many changes over the past century and its etiopathology remains ever elusive [1]. Schizophrenia is a chronic, severe, and disabling brain disorder that has affected people throughout history. About 1 percent of Americans have this illness [2]. The symptoms of schizophrenia may be divided into the following four domains: positive symptoms psychotic symptoms, such as hallucinations, which are usually auditory; delusions; and disorganized speech and behavior. Negative symptoms - decrease in emotional range, poverty of speech, and loss of interests and drive; the person with schizophrenia has tremendous inertia. Cognitive symptoms - neurocognitive deficits (eg, deficits in working memory and attention and in executive functions, such as the ability to organize and abstract); patients also find it difficult to understand nuances and subtleties of interpersonal cues and relationships. Mood symptoms-patients often seem cheerful or sad in a way that is difficult to understand; they often are depressed [3].

Delusions are false fixed beliefs that are out of touch with reality. They are held with total conviction and cannot be altered by the presentation of facts or by appeal to logic or reason [4]. Several types of delusions exist. These include delusions of grandeur (belief of exaggerated importance), persecutory delusions (belief of deliberate harassment and persecution), reference delusions (belief that the thoughts and behavior of others are directed towards oneself) and somatic delusions (belief that part of the body is diseased, distorted or missing) [3]. Among these, somatic delusions in schizophrenic patients are particularly understudied and under reported. Delusional disorders are thought to be stable in their course and less than $25 \%$ progress to schizophrenia [5]. Impaired or altered processing of perceptual disturbances is common in people suffering from psychotic disorders [6]. Although studies have found a genetic predisposition in those suffering from schizophrenia spectrum disorders and have shown a familial inheritance pattern the same is not true for delusional disorders [5].

Following is a case report of a Hispanic middle-aged woman with a long psychiatric history of schizophrenia who also presents with various complex and bizarre somatic delusions evidenced by her presenting complaints and numerous emergency room visits.

\section{Case Report}

The patient was a poor historian and was unable to recount what occurred immediately prior to her admission. According to patient care team at her residence, about two weeks prior to admission, she had run out of food stamps and there was some difficulty in renewing them. She became agitated, hostile and aggressive towards people at her residence and reported to her social worker that she felt like committing suicide and homicide. Emergency Medical Services (EMS) was activated and she was taken to the psychiatric emergency room. Upon evaluation, she was observed to be expansive, internally preoccupied reporting nonspecific homicidal and suicidal thoughts. She was subsequently admitted to the inpatient unit to treat her acute presentation. While on the inpatient unit, she denied suicidal or homicidal ideation, but was

*Corresponding author: Panagiota Korenis, Department of Psychiatry, Albert Einstein College of Medicine-Bronx Lebanon Hospital Center, 1276 Fulton Avenue, Bronx, New York, NY, USA, Tel: 914-426-5127; E-mail: korenismd@gmail.com

Received March 15, 2015; Accepted April 21, 2015; Published April 30, 2015

Citation: Patel R, Gonzalez L, Joelson A, Korenis P (2015) Schizophrenia with Somatic Delusions: A Case Report. J Psychiatry 18: 290 doi: 10.4172/2378-5756.1000290

Copyright: $\odot 2015$ Patel R, et al. This is an open-access article distributed under the terms of the Creative Commons Attribution License, which permits unrestricted use, distribution, and reproduction in any medium, provided the original author and source are credited 
observed to be responding to internal stimuli and reported auditory hallucinations. She stated that she would hear anywhere from ten to fifteen different voices, both male and female, that were constantly fighting with each other and talking about her. When asked to elaborate what the voices would say, the patient responded: "stupid stuff" and would not elaborate further. She denied the use of drugs or alcohol, and her urine toxicology was negative on admission.

Throughout the course of her admission she was noted to verbalize several somatic complaints some of which were exaggerated, unrealistic and bizarre. Specifically she reported that her bone was "sticking out" and that her wrist was twisted. She was further convinced that her arm was dysfunctional as the veins in her arm were not going in the "right direction." Of note, she would wince of pain when her arm was examined, but when she was distracted and her arm was touched, she did not react. An X-ray taken after the onset of this complaint was negative. She became frustrated as her continued attempts for a cast were denied as there was no indication, and eventually stated "I just need a cast, I keep saying it. Nobody pays attention to me. You know what I'm going to do? One day, I'm just going to say 'My arm just fell down on the floor. I am dying' but it doesn't matter, because I don't have a cast." When asked why she felt like she was dying she replied "I could die of it" and that her broken arm gave her fever and chills. The patient exhibited many somatic delusions in various other parts of her body. After she was hit by an orange following a verbal altercation with another patient, she described the pain as it "feels like my head was cracked open," and believed that she had a huge bump on her forehead, which she did not. The patient also described a pain in her right leg after reporting that she fell down some stairs about two years ago. She felt that her leg was broken and that the two bones in her lower leg were "broken and twisted," yet had no difficulty ambulating and exam did not indicate any abnormality. After getting her last intramuscular injection of Invega Sustenna prior to discharge, the patient reported that she felt like "all my little tendons and ligaments are like, all standing up" and if "[the injection] would had been placed in my bone, I wouldn't have a bone because it would've been a broken arm." At one point, the patient stated that she felt as if she had an abortion because her "stomach got big and hard and then got soft and small." She continued by saying that she did not want to go to the hospital to the gynecologist because they are going to "hospitalize me and induce the labor and I'm not due yet." A beta-HCG test on admission was negative. When told that she was not pregnant, the patient replied: "Well they will induce it." She went on to reiterate that she felt as if she had an abortion and it then "grew back." The patient was noted to still be hearing voices on and off and her dose of antipsychotic was thus increased. Her third week on the unit, the patient began to show some improvement, continued compliance with her medication, but still remained internally preoccupied and preoccupied with various somatic delusions.

\section{Previous Medical ER Visits and Hospitalizations}

According to her records, between July of 2009 and March of 2014 the patient had 27 emergency room visits and three brief inpatient medical admissions. Over that time period she visited five different New York City hospitals requesting treatment for extremity pain/ myalgia, unspecified chest pain, Gastroesophgeal Reflux Disease (GERD)/dysphagia, and abdominal pain/gastritis. Her three inpatient admissions were twice for esophageal reflux, and once for "other chest pain." These complaints are in parallel with many of the patient's complaints during her most recent psychiatric hospitalization.

\section{Discussion}

\section{Importance of ruling out medical co-morbidities schizophrenic patients with somatic delusions}

Medicine in $21^{\text {st }}$ century presents with numerous challenges to the physician including an aging population, increased survival rate of chronic medical illnesses and widespread substance abuse. Clinicians are more likely to encounter psychiatric conditions that are associated with medical co-morbidities or secondary to medical illness. Studies have shown that estimates of co-morbid medical illness in psychiatric patients ranges from $4 \%$ to $18 \%$ [7]. In the Emergency Room (ER) setting, it is very crucial to rule out possible medical causes for patients presenting with psychiatric symptoms. A wide range of medical conditions and treatments may result in abnormal behavior, and many medical disorders may produce or exacerbate psychiatric symptoms in patients with pre-existing mental illness. Failure to detect and diagnose underlying medical disorders may result in significant and unnecessary morbidity and mortality. All patients presenting with new psychiatric symptoms need thorough medical evaluations. Patients with known histories of psychiatric illness are at greatest risk for having a medical condition overlooked because clinicians too often presume that these patients' abnormal behavior is caused by their psychiatric illness. Psychiatric patients who present to the ER setting require a thorough medical evaluation including all necessary diagnostic work up. In other settings, including the inpatient psychiatric unit, the medical knowledge and skills of the psychiatrist are required as to not miss potential medical emergencies when immediate medical assistance is not available [7].

\section{Why do patients present with somatic delusions?}

Review of literature suggests numerous reasons why people may present with somatic delusions. One hypothesis is that it serves as a defense mechanism to cope with life stressors including coping with psychological trauma. In the case of our patient described above, prior to her admission she had numerous financial and environmental stressors as well as a history of physical abuse as a child by her mother. In addition, somatic delusions may be a manifestation of factitious disorder whereby the patient has an unconscious desire to assume the "sick role."

\section{Cultural Impact}

A small 1987 study by Escobar studied the prevalence of somatic symptoms in a schizophrenic population of both non-Hispanic white and patients of Hispanic descent. The diagnostic tool used was the Diagnostic Interview Schedule (DIS), which consists of 37 different somatic symptoms with multiple questions pertaining to each one. In the Hispanic group of patients, 35\% exhibited more than 4 items, whereas in the non-Hispanic White group, only 7\% experienced that amount of somatization traits. Interestingly, "heart beating fast" and "shortness of breath" were two of the symptoms most commonly experienced by Hispanic patients ( 31 and 26.2 percent, respectively) [8]. This relates back to our patient who visited the emergency room three times for "nonspecified chest pain" in the month leading up to her most recent hospitalization. Furthermore, in a 2000 article by Weisman, et al., it was suggested that manifestations of psychological disease depends on the religious and cultural beliefs of a given population. Patients of Hispanic decent tend to externalize psychiatric problems and attribute them to physical problems. It is easy to understand how somatic delusions emerge in patients for whom it is difficult to accept illness that stems solely from the mind. 
- Always a therapeutic challenge

- A strong therapeutic alliance with one provider is paramount

- Acknowledgement of delusions, though non-confrontationally

- Treat substance use/abuse

- Stable relationships and adequate social supports are necessary

Table 1: Management and treatment of somatic delusions.

\section{Management and Treatment of Somatic Delusions}

Those who present with somatic delusions pose many therapeutic challenges to the treating clinician. Generally, resistance to engage in treatment is a common obstacle for clinicians working in mental health. It becomes far more challenging when the patient exhibits symptoms that are ego syntonic-in accordance with the desires of the ego-as is the case with the patient described above. Developing a therapeutic alliance with the patient and having them accept psychiatric treatment is by far one of the biggest initial challenges. One strategy in the management of somatic delusions involves the acknowledgment of the delusion, but for it to even be remotely successful there must exist a therapeutic alliance and the patient must be confronted in a non-confrontational manner in order to preserve this alliance. For those with somatic presentations, consolidation of treatment to one provider and increasing the number of visits is recommended. A reduction in the frequency of any selfinjurious behaviors as well as diminishing substance use can help in the management as well. Ensuring that those experiencing somatic delusions have stable relationships and adequate social supports are also imperative for the success of treatment (Table 1).

\section{Conclusion}

In conclusion, schizophrenia with somatic delusions is an understudied and under reported phenomenon that is poorly understood in the medical literature. It poses plentiful challenges to the treatment provider. The role of the psychiatrist in assessing and managing these patients is decisive and requires significant investigation and attention to detail. In the clinical setting, the psychiatrist is faced with the obstacle of developing a therapeutic alliance to engage the patient in treatment and rule out potential medical complications and co-morbidities. Further exploration and investigation into schizophrenia with somatic delusions is necessary to better understand its psychiatric manifestations and consequences.

\section{References}

1. Haller CS, Padmanabhan JL, Lizano P, Torous J, Keshavan M (2014) Recent advances in understanding schizophrenia. F1000Prime Rep 8 6: 57.

2. National institute of mental health, schizophrenia booklet.

3. (5thedn). Arlington: American Psychiatric Association; 2013. American Psychiatric Association: Diagnostic and Statistical Manual of Mental Disorders

4. Centre for Mental Health (2001) Mental health for emergency departments-A reference guide. Gladesville: Better Health Centre for NSW Health.

5. Issa BA (2010) Delusional disorder-somatic type (or body dysmorphic disorder) and schizophrenia: a case report. Afr J Psychiatry (Johannesbg) 13: 61-3.

6. Reeves RR, Torres RA (2003) Exacerbation of psychosis by misinterpretation of physical symptoms. South Med J 96: 702-4.

7. Lagomasino I, Daly R, Stoudemire A (1999) Medical assessment of patients presenting with psychiatric symptoms in the emergency setting. Psychiatr Clin North Am 22: 819-50.

8. Escobar JI (1987) Cross-cultural aspects of the somatization trait. Hosp Community Psychiatry 38: 174-80. 